\title{
Primary Cardiac Fibroma and Cardiac Conduction System Alterations in a Case of Sudden Death of a 4-month-old Infant
}

\author{
Donatella Mecchia*, Anna Maria Lavezzi and Luigi Matturri
}

"Lino Rossi" Research Center for the study and prevention of the unexpected perinatal death and the sudden infant death syndrome (SIDS), University of Milan, Milan, Italy

\begin{abstract}
A 4-month-old female infant considered to be in good health died suddenly and unexpectedly. Post- mortem examination was requested, with clinical diagnosis of sudden infant death syndrome. At autopsy the infant was described in good health. Histo- logical examination of the heart found a cardiac fibroma compressing the atrio-ventricular node and the examination of the cardiac conduction system showed an accessory fiber of Mahaim (nodo-ventricular) and cartilaginous metaplasia of the cardiac fibrous body. Probably the concomitant presence of cardiac conduction system abnormalities and a septal fibroma, compressing the atrio-ventricular node, could have an important role in causing the sudden death.
\end{abstract}

Keywords: SIDS, Primary cardiac fibroma, cardiac conduction system.

\section{INTRODUCTION}

Primary cardiac tumors are very rare. In infancy and childhood they are even more infrequent, with a prevalence of 0.0017 to 0.28 in autopsy series. In contrast, the incidence during fetal life is approximately $0.14 \%$ [1-3].

Cardiac fibroma is the second most common childhood cardiac tumor $[4,5]$. It tipically originates from the ventricular septum or ventricular free wall. Although cardiac fibroma is histologically benign and does not metastasize or infiltrate, it can cause hemodynamic and conductive abnormalities resulting in sudden death [1].

This report will focus on the histological examination of the heart and conduction system in a case of sudden death in a 4-month-old infant.

\section{CASE REPORT}

The infant was a female 4-months-old, born at 37 weeks of gestation, with a birth weight of $2270 \mathrm{~g}$ (low birthweight, at the $5^{\text {th }}$ percentile). She was in apparent good health until the day she died, suddenly and unexpectedly. Her parents said that they knew of no illness or complaint of any nature, except for two episodes of vomit two days before. There was no significant family history relevant to the case. In particular, there was no familiarity for tuberous sclerosis and Gorlin's syndrome (multiple nevoid basal carcinomas, jaw cysts, and bifid ribs), both associated with cardiac fibromas [1].

\footnotetext{
*Address correspondence to this author at the "Lino Rossi" Research Center for the study and prevention of the unexpected perinatal death and the sudden infant death syndrome (SIDS), University of Milan, Milan, Italy; Tel: +39-02-503.208.04; Fax: +39-02-503.208.23;

E-mail: donatella.mecchia@unimi.it
}

A complete autopsy was performed, according to the autopsy protocol usually followed by our center in cases of sudden death [6]. Multiple samples of all organs were fixed in $10 \%$ formalin buffer, processed, and embedded in paraffin. Five-micrometer sections were stained with hematoxylin and eosin. The heart had been regularly examined for pathological changes in the atria, septa, ventricules, pericardium, endocardium, and coronary arteries. Samples of the myocardium were stained with hematoxylin and eosin and trichromic Heidenhain (azan). Histological observations were focused on the cardiac conduction system, which was removed in two blocks, according to our guidelines [6]: the first included the sino-atrial node (SAN) and the "crista terminalis," the second contained the atrioventricular node (AVN), His bundle, and bundle branches. The samples were stained alternately with hematoxylin and eosin and trichromic Heidenhain (azan).

At autopsy, the infant was described in good health, with body length and weight at the $5^{\text {th }}$ percentile. There were no marks of violence. The lungs were congested and slightly edematous. The heart, arrived without the tip, weighed $25 \mathrm{~g}$; the cardiac diameters were as follows: transverse $3.5 \mathrm{~cm}$ and anteroposterior $2.9 \mathrm{~cm}$. We could not measure the longitudinal diameter due to the absence of the tip. The myocardium was brownish and homogeneous in appearance, without any evident alteration. The foramen of Botallo was open for about $3 \mathrm{~mm}$. The coronaries appeared normal.

Histological examination of the atrio-ventricular node showed an accessory fiber of Mahaim (nodo-ventricular), cartilaginous metaplasia of the cardiac fibrous body and preatherosclerotic lesions of the artery of the atrio-ventricular node (Fig. 1). It was also present a non-encapsulated proliferation of fibroblasts with regular nuclei and an abundant network of collagen and elastic fibers that dissociated and entrapped the myocytes (Fig. 2). These morphological fea- 
tures led to the diagnosis of primary cardiac fibroma compressing the atrio-ventricular node [5]. Mitotic figures and cytologic atypia were not observed. No foci of dystrophic calcification were present. The SAN, adjacent nerve plexuses, and internodal right atrial myocardium were free of alterations.

\section{DISCUSSION AND CONCLUSIONS}

Sudden infant death syndrome affects about 0.7-1 per 1000 live births [7]. The most accredited pathogenetic hypothesis of SIDS (cardiac-arrhythmogenic, respiratory-apnea and/or suffocation and dyskinetic-visceral) seem to give preference, in most cases, to a dysfunction of the autonomic nervous system which control the cardio-respiratory activity, the first upper digestive tract and the awakening [8-10].

Although the diagnosis of SIDS and sudden unexplained perinatal death is by exclusion, changes of the cardiac conduction system and brainstem are of great value in establishing and characterizing these forms of death [11-13]. Although the majority of these fatalities are related to sudden infant death syndrome (SIDS), a very small percentage is due to primary cardiac neoplasm [11]. This is the second case of fibroma of the fibrous trigone found in a series of 69 cases of death examined.

Cardiac fibroma is a rare tumor in pediatric practice but, after rhabdomyoma, it is the second most frequent tumor of childhood in autopsy series [3-5]. Fibromas are usually large and intramural, occupying the septum and/or the anterior wall of the left ventricle. The tumor is composed of fibroblasts, dense connective tissue, and a small admixture of myocardial cells [5]. Intramural cardiac tumors are frequently associated with disorders of the rhythm, particularly in children [8]. In our case the neoplasm was adjacent and compressing the atrio-ventricular node. In the cases described in literature were reported significant alterations of the rhythm $[1,5,14]$. In our case the tumor was clinically silent.

Sudden infant death syndrome (SIDS) "gray zone" or borderline cases are defined as those cases in which it is difficult to establish whether the pathological findings are really cause of death $[15,16]$. Krous et al. described as "unclassified sudden infant deaths" those cases that do not meet the criteria for a diagnosis of SIDS [17]. In fact, according to the original definition, SIDS is "... the sudden death of any infant or young child, which is unexpected by history, and in which a thorough post-mortem examination fails to demonstrate an adequate cause for death” [18]. In 1991 the National Institute of Child Health and Human Development (NICHHD) described more precisely inclusion and exclusion criteria for SIDS [19], but it is still not present a clear classification of these deaths.

In our case, the concomitant presence of cardiac conduction system abnormalities (an accessory fiber of Mahaim and cartilaginous metaplasia of the cardiac fibrous body) and a septal fibroma, compressing the atrio-ventricular node, could have an important role in causing the sudden death.

Infact, the circum- stances that the patient died suddenly and unexpectedly and that a fibroma was found compressing

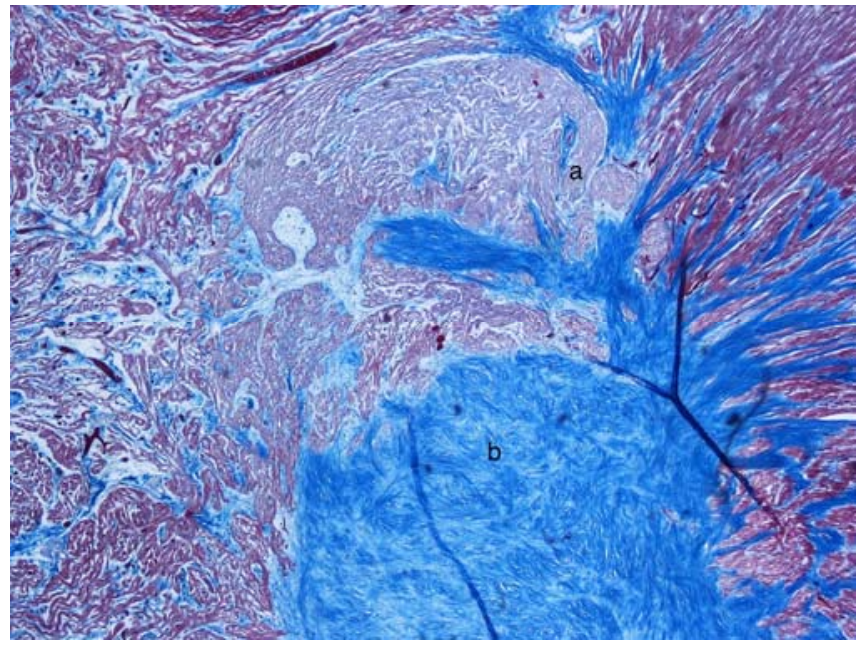

Fig. (1). a) Fiber of Mahaim (nodo-ventricular); b) Cartilaginous metaplasia of the central fibrous body. Azan stain, $40 \mathrm{x}$.

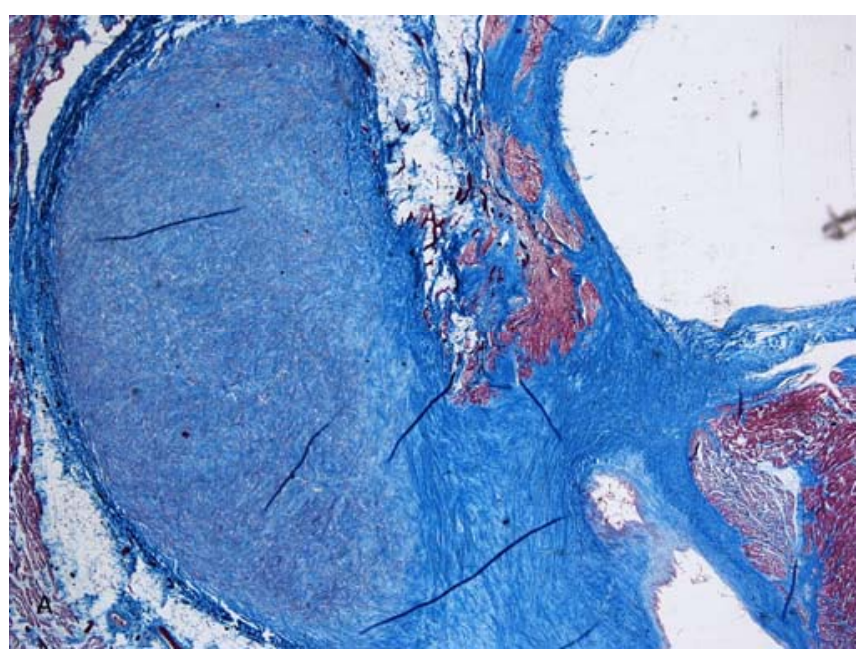

A

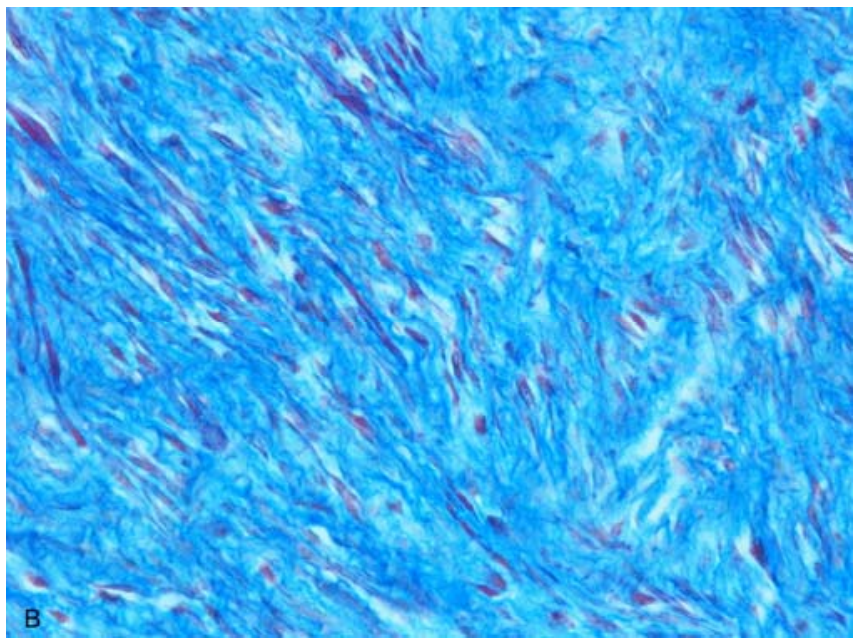

B

Fig. (2). Cardiac fibroma. Non-encapsulated proliferation of fibroblasts with regular nuclei and an abundant network of collagen and elastic fibers that dissociated and entrapped the myocytes. A) Azan stain, $20 \mathrm{x}$; B) Azan stain, $60 \mathrm{x}$. 
the atrioventricular pathway suggest that the two facts could be causally related.

The anatomic abnormalities observed in the present case concur with a diagnosis of SIDS/sudden unexplained death gray zone, without any proven sequential occurrence.

\section{CONFLICT OF INTEREST}

The authors confirm that this article content has no conflicts of interest.

\section{ACKNOWLEDGEMENTS}

None declared.

\section{REFERENCES}

[1] Yamaguchi M, Hosokawa Y, Ohashi H, Imai M, Oshima Y, Minamiji K. Cardiac fibroma: long term fate after excision. J Thorac Cardiovasc Surg 1992;103:140-5.

[2] Holley DG, Martin GR, Brenner JL. Diagnosis and management of fetal cardiac tumors: a multicenter experience and review of published reports. J Am Coll Cardiol 1995; 26: 516-20.

[3] Uzun O, Wilson DJ, Vujanic GM, Parsons JM, De Giovanni JV. Cardiac tumors in children. Orphanet J Rare Dis 2007, 2: 11.

[4] Basso C, Valente M, Poletti A, et al. Surgical pathology of primary cardiac and pericardial tumors. Eur J Cardiothorac Surg 1997; 12: 730-8.

[5] Burke A, Virmani R. Benign tumors of fibrous tissue. In: Rosai J, Sobin LH, Eds. Tumors of the Heart and Great Vessels, Atlas of Tumor Pathology. 3rd series. Washington, DC: Armed Force Institute 1996; vol. 16: pp. 69-77.

[6] Matturri L, Ottaviani G, Lavezzi AM. Guidelines for neuropathologic diagnostics of perinatal unexpected loss and sudden infant death syndrome (SIDS)- A technical protocol. Virchows Arch 2008; 452: 19-25.

[7] Neonatal and perinatal mortality: country, regional and global estimates. World Health Organization (WHO) 2006. http://www.who.int/making_pregnancy_safer/publications/neonatal .pdf. Available from: [Accessed July 21, 2006.]
[8] Rossi L, Matturri L. Cardiac conduction and nervous system in health disease and sudden death: an anatomoclinical overview. Osp Maggiore 1995, 89: 239-57.

[9] Guntheroth WG, Spiers PS. Are bedding and rebreathing suffocation a cause of SIDS? Pediatr Pulm 1996; 22: 335-41.

[10] Matturri L, Biondo B, Suarez- Mier MP, Rossi L. Brain stem lesions in the sudden infant death syndrome: variability in the hypoplasia of the arcuate nucleus. Acta Neuropathol 2002, 194: 1220.

[11] Matturri L, Ottaviani G, Ramos SG, Rossi L. Sudden infant death syndrome (SIDS): a study of cardiac conduction system. Cardiovasc Pathol 2000 9: 137-45.

[12] Ottaviani G, Matturri L, Rossi L, James TN. Crib death: further support for the concept of fatal cardiac electrical instability as the final common pathway. Int J Cardiol 2003; 92: 17-26.

[13] Ottaviani G, Matturri L, Mingrone R, Lavezzi AM. Hypoplasia and neuronal immaturity of the hypoglossal nucleus in sudden infant death. J Clin Pathol 2006;59:497-500.

[14] Kelmanson IA. Relationship between the incidence of sudden infant death syndrome (SIDS) and other causes of infant mortality in the industrialised countries. Paediatr Perinat Epidemiol 1994; 8: 166-72.

[15] Ottaviani G, Matturri L, Bruni B, Lavezzi AM. Sudden infant death syndrome "gray zone" disclosed only by a study of the brain stem on serial sections. J Perinat Med 2005; 33: 165-9.

[16] Ottaviani G, Matturri L. Fibromuscular hyperplasia of the pulmonary artery in sudden infant and perinatal unexpected death. 8th World Congress of Perinatal Medicine. September 9-13th, 2007, Florence, Italy. J Perinat Med 2007; 35(Suppl): 229.

[17] Krous HF, Beckwith JB, Byard RW, et al. Sudden infant death syndrome and unclassified sudden infant deaths: a definitional and diagnostic approach. Pediatrics 2004; 114: 234-8.

[18] Beckwith JB. Discussion of terminology and definition of the sudden infant death syndrome. In: Bergman AB, Beckwith JB, Ray CG (Eds) Sudden infant death syndrome. Proceedings of the Second International Conference on the Causes of the Sudden Death in Infants. Seattle 1970; University of Washington Press, pp 14-22.

[19] Willinger M, James LS, Catz C. Defining the sudden infant death syndrome (SIDS): deliberations of an expert panel convened by the National Institute of Child Health and Human Development. Pediatr Pathol 1991: 11: 677-84.

(C) Mecchia et al.; Licensee Bentham Open.

This is an open access article licensed under the terms of the Creative Commons Attribution Non-Commercial License (http://creativecommons.org/licenses/ by-nc/3.0/) which permits unrestricted, non-commercial use, distribution and reproduction in any medium, provided the work is properly cited. 